\title{
CATEGORIZATION OF CLASHES AND THEIR IMPACTS ON CONSTRUCTION PROJECTS
}

\author{
Petr Matejka, Daniel Sabart \\ Czech Technical University in Prague, Czech Republic \\ petr.matejka@fsv.cvut.cz,daniel.sabart@fsv.cvut.cz
}

\begin{abstract}
The paper deals with problematics of clashes in construction projects. In the first part the general definition of a clash is described along with its origin, brief history and development in built environment and construction management using Building Information Modeling (BIM). The second part of the paper focuses on detailed categorization of clashes. Each category is based on its specific characteristics and algorithms of its detection process - Hard clashes are exclusively geometrically oriented, Soft clashes include implementation of a static buffer, 4D clashes are based on errors related to project schedule and Clashes of animated objects utilize dynamic buffer. Practical examples are also provided. In the third part, the impact of clashes on a project in construction industry is discussed. This offers an overview of how important collision control system is and why managers should focus on it. The relevancy of clashes is also interpreted by data achieved from publications and reports from actual projects. Next part of this paper deals with the problematics of resolving the clashes. It mentions different approaches of clash resolving and emphasizes important requirements for their proper application. Based on the conducted research, simple formulas, which could be used for quantifying efficiency of computer clash detection and for comparison with manual clash detection, are proposed. The final part of the paper contains conclusions and discussion about the issues described in the text of the paper.
\end{abstract}

Keywords: BIM, clash detection, construction, efficiency, P-T-C analysis.

\section{Introduction}

In this paper a topic of clashes in digital buildings models is explained. The first part of the paper describes a general definition of a clash, its history and gradual implementation in construction management through information modeling. In the second part, the paper proposes clashes taxonomy for future use. Each of the clash categories is being analysed in detail and examples are listed. In the third part of the paper, potential impacts of clashes are described. Practical reports about the relevancy of clashes and utilization of information models in real projects are included. The next part focuses on a methodology of resolving the clashes, based on practical experience of the authors. The last part of the paper presents simple formulas, which could be used for quantification of clash detection efficiency. Practical application of formulas is also presented for the purpose of their explanation.

\subsection{Clash detection overview}

The original idea of detecting the object spatial conflicts emerged in the entertainment industry. During programming the first computer games, developers suddenly faced an entirely new problem. They realized the inevitability of collision of solid elements while modeling their trails. Therefore, they had to define what to do, when two entities touch each other. It practically led to a development of a new program code that should define the general behaviour of every clash-involved object. The basic approach was to inspect whether two objects collide in three dimensional space. Due to dynamic characteristics of computer games, it was necessary to consider the positioning of objects in time. The solution was in introduction of certain object attributes and compilation of behaviour rules in case that the system detects a collision with another entity.

Due to rapid development of information technologies, civil engineers started to deal with such problems when using BIM (Building Information Modelling). Built environment adopted some approaches to develop automated clash detection and indication of problematic elements. This BIM clash detection system evolved significantly as time went on. Currently, the research is continuously developing tools that are more sophisticated. Such tools aim to help in activities such as model processing and general design making.

\section{Clash types}

As it was mentioned, clash emerges in the case of spatial and time collision of two or more entities. Because of that, the most difficult part of the design phase is proper element positioning and 
construction work planning. The most relevant information included in the BIM model is therefore geometrical and time data. It is possible to sort clashes in four types as follows:

1. hard Clashes;

2. soft Clashes;

3. 4D Clashes $[1 ; 2]$;

4. animated clashes (clashes of animated objects).

The paper proposes this taxonomy of clashes for future use.

\subsection{Hard Clashes}

Hard clash is a type of a conflict, which involves only geometrical issues. These basically result in mutual intersection with no specified joint or opening. Hard clashes are probably the most easily recognizable of all clash types, because of both logical arrangement of elements and their null dynamics. These entities are considered to be static and therefore it is possible to expect their permanent installation and adequate interaction with the rest of structure. Hard clashes are, for example, collision of piping and a wall without definition of the opening or staircase colliding with horizontal structures such as beams, floor, etc. Other frequent modeling faults leading to hard clashes are, for example, bad connection between the partition and the wall or placing the toilet too close to the door. Collisions of static and MEP models are one of the most commonly identified issues in the design.

This category is considered to be crucial and it is vital to deal with it as such. Because of its high obstacle potential, it is important to handle every occurrence of hard clash as soon as possible. It is emphatically advised to focus on quick elimination of such issue. The system of authorized engineers and architects helps deal with collisions already in the design making process. The majority of clashes is therefore solved on time and does not threaten the next phases of the project. However, there might be exceptions caused by lack of practice, insufficient professionalism of engineers, bad coordination of professions or just thoughtlessness and lax approach of any involved worker.

There is a special category of hard clashes, which contain duplicates. During the 3D modeling it may happen that the user accidentally put two same objects in one place. Contemporary modeling software will usually highlight the occurrence of the duplicate. This feature does not have to work properly all the time and because of that two-fold objects might still occur in the model. These collisions are basically hard clashes of two or more exactly the same elements occupying exactly the same place. Therefore, it is quite easy to detect them by the definition of hard clashes. Despite of relative ease of their detection, duplicates can lead to significant inaccuracies, e.g., in bills of quantities.

\subsection{Soft Clashes}

Soft clashes (sometimes called clearances) are defined not only by geometrical data. In this case space, connection, installation and manipulation requirements are considered in the control process. The word buffer is sometimes being used to define these demands. It is generally a space around an object with certain requirements. Buffers of entities involved in soft clashes are static in contrast with dynamic ones in clashes of animated objects. According to Reddy, "Soft clash generally highlights the interference between the system and the buffer which is set around another system." [3]

It is possible to define the following collisions as soft clashes, for example: insufficient beam or lintel positioning, placing the component (column, wall, etc.) right in front of a window, door or opening, lack of space between fixtures or inadequate width of a stair landing. Other design errors defined as soft clashes are, for example, insufficient dimensions of various stair components or manipulation space in front of BMS (Building Management System) objects, fire extinguishers, etc. Inspection of a minimum headroom in both rooms and stairs belongs also to the soft clashes checking system.

Commonly, soft clashes are being inspected because of the demand for the efficiency of a space utilization. Used algorithm is based on certain rules, which define general correctness of a design from a safety and proper using point of view. In contrast to hard clashes, these collisions do not have to necessarily reflect critical design errors, due to which it would not be possible to finish or use the 
property. Sometimes it is possible to tolerate soft clashes at the expense of subsequent restrictions in further using. This option should be chosen only in the case, when the error is of relatively low importance, while both expense for its elimination and caused delay are inadequately high. Because of such complicated approach, it is quite important to ensure a proper human-based control system as it is considered to bring better results and enhancement to general practice.

\subsection{D Clashes}

Engineers can encounter problems caused by differing form the schedule or coordination plans that can significantly affect ongoing works. These problems are inspected through 4D clashes control system. 4D collisions basically occur during the construction and are not directly associated with the structure design. 4D clashes are, for example, insufficient distance between the crane and the structure, stored material accumulation, poor design of a building site and its facilities considering manipulation space for lorries and heavy machinery, the onset of the work crew on a site, where the material is not yet prepared, the onset of the work crew on a site, where another crew is still working, or conflict between the crane and recently erected scaffolding.

The common cause of some 4D clashes is a poor quality of construction management. However, tools that managers can use for conflict prediction have preventive function only. It is not possible to fully count on their outcomes alone and therefore it is essential to ensure appropriate on-site adoption of provided suggestions. Nevertheless, 4D collision checking system can be used to improve planning the building site and site facilities. In the case of placing the virtual crane too close to the structure, the system should alert the user. This can lead to optimization of associated designs. Using certain rules might be complicated due to variability of site and construction conditions. It is recommended to modify the rules considering future development.

\subsection{Animated clashes}

Collisions belonging to this category are being identified on the base of their dynamic characteristics. This involves animating the objects, representing, for example, people walking on stairs and through corridors, or components that require manipulation space. This type of clashes is practically based on soft clashes with the difference in using rather dynamic buffer than the static one.

Animated clash can occur when moving a mobile crane from a former non-collision position to a new one generating conflict with a structure. Another example is moving a virtual person through corridors and stairs to inspect correction of prescribed dimensions. Appropriate algorithm of defining the dynamic buffer should be different and presumably more difficult than static buffer used in soft clashes. Animated clashes also require more modelling work and are not utilized as much as previous clash categories.

\section{Impact of clashes}

As it has been discussed before, collisions represent potential threats. The benefit of using the BIM model is a function of simulating the construction process and installation of individual components. This approach offers an opportunity to prepare for future risks caused by design errors in advance.

According to Kymmel, clash detection is the most efficient tool for virtual work coordination and it should be integrated to the BIM model processing work-flow [4]. Similarly, Reddy states that clash detection is basic, but extremely valuable use of the BIM model processing technology. The author does not fully exclude the option of operative clash solution but he highlights its negative impact on both financing the project and keeping up with the schedule [3].

It is commonly recommended to deal with collisions and similar deficiencies of the design as soon as possible. If the clash is not solved in one of the initial phases, it will physically appear on the site, which would lead to changes in the projects and befitting delays [3]. Ignoring of important clashes generally brings almost certain increase in construction cost due to associated repairs and rework [5].

There are many examples of construction companies reporting significant savings thanks to using BIM in clash detection. According to the BAM company, the BIM clash detection system saved about $350000 \mathrm{GBP}$ on the Leeds Arena project in Great Britain by early identification of errors and 
therefore foreseeing the threats [6]. Similarly, Holder Construction has announced the savings of 135000 USD thanks to clash detection with just 4000 USD investments in BIM tools [7]. TesseractDesign published cost estimation of reworking a single ignored clash that has reached 750 USD [8]. The authors also state savings of 9000 USD thanks to clash detection on a project of a multifunctional building. With clash detection software coordination investments of 2945 USD they also state the ROI (Return On Investment) of $306 \%$. On a different and bigger project, they mention total expense of 43402 USD, savings of 127850 USD and ROI of $295 \%$. The authors therefore speak about $300 \%$ of investment returns concerning the BIM clash detection system [8].

Nowadays, the BIM methodology is being slowly adopted by HSE (Health And Safety). Ignoring the clashes that seem to be of low relevance might lead to dangerous situations and risking injury or even death. In the USA, engineers have developed a system of GPS transmitters connected with a BIM model. These transmitters, which are sewn in safety vests, should alarm workers, when they enter a forbidden or dangerous zone [9]. This provides a tool for supervising the safety of workers in a new way.

\section{Resolving clashes}

Clashes can appear in different project stages. Some of them can be resolved in the starting phase, which is considered to be the most economical approach. Especially during refurbishing projects is the occurrence of an originally undetectable clash quite common. Such conflicts must be solved operatively. In many cases it is possible on-site only sometimes supported by information technology. The idea of many software companies is to develop a virtual inspection system using the BIM model via tablet computers. This should be a tool for responsible workers, who can run a visual test and quickly resolve identified conflicts. Such a control system offers a good opportunity to accelerate resolving errors in the design, which have been omitted.

Traditional method of detecting clashes (especially hard and soft ones) is overlaying individual drawings on a light table - i.e. HVAC (Heating Ventilation And Cooling) versus architectonical design drawings. Compared to the software-based approach the traditional method is tedious and faulty [10]. Collisions are being solved also during the construction work, but it is important to properly merge and coordinate all activities. Architect or construction design planner has to take into account all objects appearing both before and during the construction works. From the ideal design point of view, it is necessary to consider the objects that will appear after the project completion and are added by a final user. This includes, for example, a design of a living room layout - the architect must take care of spatial requirements of commonly used objects such as the dining table, chairs, etc.

During a non-BIM way of solving a collision on a refurbish project it is recommended to follow certain rules. It is essential to measure all relevant structures right on the site. Depending on a possible impact of a collision, good knowledge of technology and construction management is usually required. Along with that, a decent imagination of planning structures is quite helpful. It is vital to know all the specifics of the project properly. If the clash is too complicated and it is not possible to solve it on site, it is recommended to stop ongoing works, take few pictures of a collision and then solve it with the help of specialized software.

\section{Efficiency calculation (P-T-C analysis)}

In this part of the paper simple formulas of comparison of human and computable clash detection were formulated. When applied, these formulas can quantify the efficiency of clash detection systems to another. The efficiency of the system is based on three coefficients (hence the P-T-C analysis). The formula was defined as follows:

$$
\eta_{r}=\frac{P \cdot T}{C},
$$

where $\eta_{r}$ - relative detection efficiency, percent;

$P$ - performance coefficient;

$T$ - preparation coefficient;

$C$ - error coefficient. 
The comparison of the two systems has to be based on the same task (i.e. same project, where there are collisions to be detected). The time used to detected collisions in the examined system is considered in the performance coefficient. The idea is to differentiate software to human factor, because there are enormous differences in collisions found.

$$
P=\frac{P_{R}}{P_{E}},
$$

where $P$ - performance coefficient;

$P_{R}$ - time necessary for detection of collisions reference (or second) system, seconds;

$P_{E}$ - time necessary for detection of defined collisions in examined system, seconds.

When software application is used to detect collisions, the overall time to conduct such operation is very short. On the other hand, it is then necessary to examine the results, because clash detection systems often identify collisions, which are not true or cannot be considered true for various reasons. This is solved by using the following formula:

$$
P_{S}=t_{d}+t_{c},
$$

where $P_{S}$ - software performance coefficient (either $P_{E}$ or $P_{R}$ );

$t_{d}-$ time used for computational clash detection analysis, seconds;

$t_{c}-$ time used for further clash analysis (results examination), seconds.

This means that, when two software applications are compared, it is still not safe to assume that $P=1$, although their time needed to detect collisions is almost the same (usually in seconds).

In case the preparation time for the task in different systems is affected by externalities or needs to be normalized (for example, when familiarity of the solver with two systems is not the same), the preparation coefficient $T$ can be used. It was defined as follows:

$$
T=\frac{T_{R}}{T_{E}},
$$

where $T$-preparation coefficient;

$T_{R}$ - time necessary for preparation of reference (or second) system, seconds;

$T_{E}-$ time necessary for preparation of examined system, seconds.

The preparation coefficient should also cover the time necessary for preparation of clash detection rules. This way it can be used for direct comparison of two software tools for addressing their user environment and setting up of the clash-detection engine.

The error coefficient is addressing the proportion between the found and existing collisions in the design as follows:

$$
C=\frac{C_{R}}{C_{E}},
$$

where $C$-error coefficient;

$C_{R}$ - number of collisions found in reference (or second) system;

$C_{E}-$ number of collisions found in examined system.

When two software applications are compared, it is not safe to assume that the same number of collisions will be found (i.e. $C=1$ ). Since the analysis is relative, it is best to consider one software application as examined and the second one as reference. Then $C_{E}$ is the amount of collisions found in the examined system, which were also found in the reference system. All excess found collisions are not considered for $C_{E}$.

In case the manual approach is compared with computational power, it is strongly advised to consider $C_{E}$ as average of at least 20 test subjects for the analysis to be accurate. It is also recommended to consider experience of human subjects and their adequate familiarity with the topic. 


\subsection{Practical application}

To explain the P-T-C analysis better, it was applied on a practical example for the purpose of this paper to compare the efficiency of the computer driven tool for clash detection with the traditional manual approach. Two model tasks were created (Project $X$ and Project $Y$ ) and for each project, about 20 experts were asked to find collisions ( 21 for Project $X$ and 22 for Project $Y$ ). Figures 1 and 2 present the scope of the design provided to the experts in Project $X$ (design for Project $Y$ was similar, a little bit more complex).

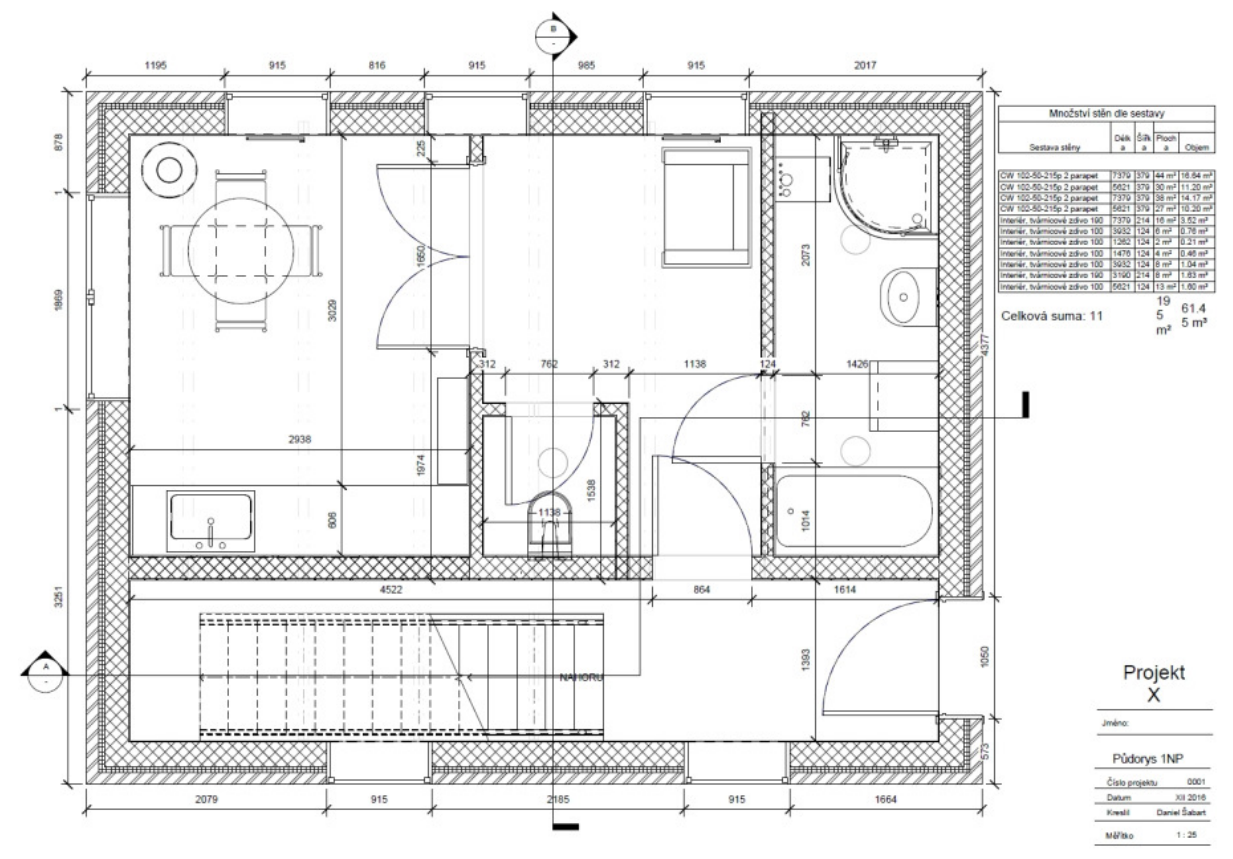

Fig. 1. Floor plan of Project $X$
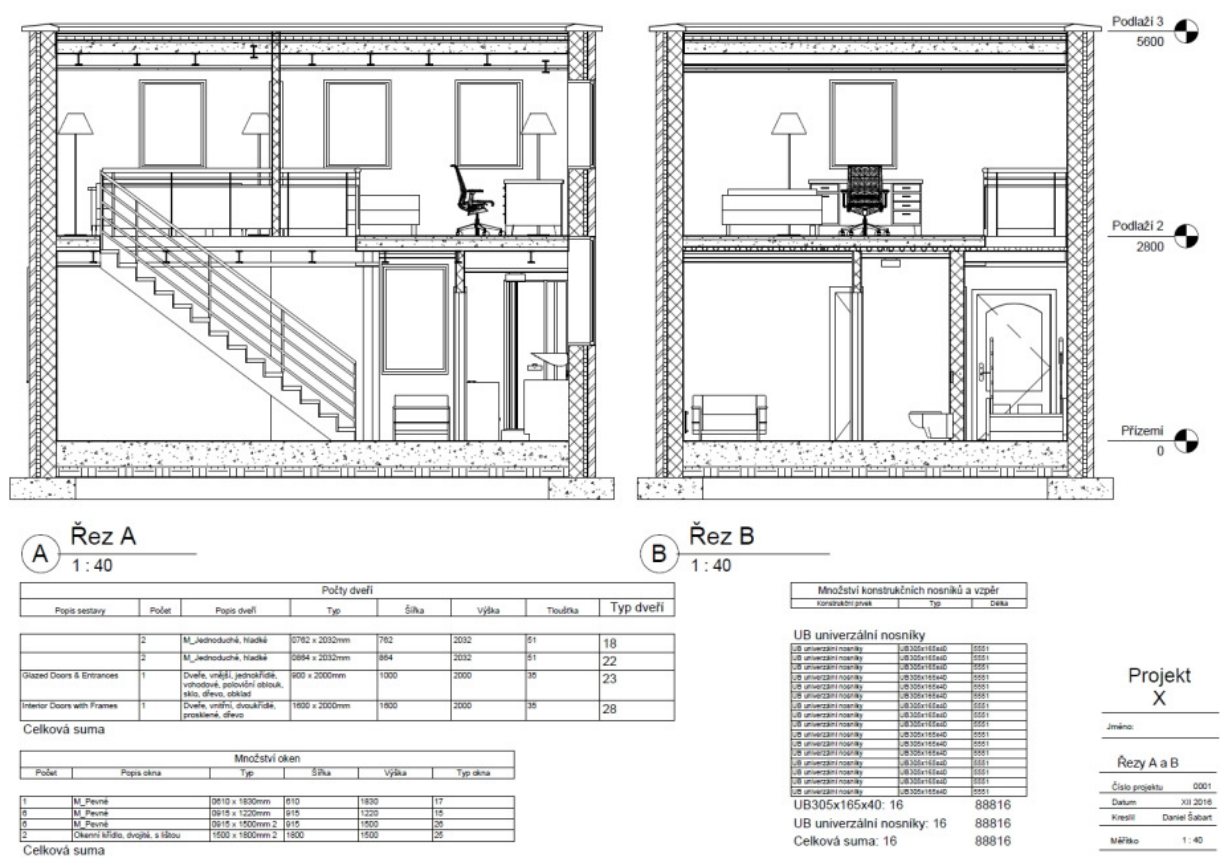

Fig. 2. Sections of Project $X$

During the test, clashes were found with the use of software. Time required for their identification and for the preparation of the software was measured. Total numbers of clashes were set and used as a reference ( 45 clashes for Project $X$ and 127 clashes for Project $Y$ ). The same was done with the experts to examine human efficiency when compared to software. The task was introduced to the expert group and they were given 7 minutes to find all collisions they can. This time was based on practical 
observation before the experiment. The expert group was able to identify average of 10,14 real clashes for Project $X$ (out of 45 identified by software) and 12.9 real clashes for Project $Y$ (out of 127 identified by software). The expert group was then given more time to match the software performance coefficient to identify total average of 16.38 collisions for Project $X$ and 18.95 collisions for Project $Y$. The efficiency was then calculated using the P-T-C analysis introduced in this paper. The results are presented in following Tables 1 and 2.

\section{P-T-C practical application for Project $X$}

Table 1

\begin{tabular}{|c|c|c|c|c|}
\hline Variable & $\boldsymbol{P}$ & $\boldsymbol{T}$ & $\boldsymbol{C}$ & Efficiency \\
\hline Reference system (Software) & 1204.15 & 300.00 & 45.00 & $100.00 \%$ \\
\hline Examined system (Human) & 420.00 & 300.00 & 10.14 & $64.62 \%$ \\
\hline Examined system (Human, more time) & 1204.15 & 300.00 & 16.38 & $36.40 \%$ \\
\hline
\end{tabular}

Table 2

\section{P-T-C practical application for Project $Y$}

\begin{tabular}{|c|c|c|c|c|}
\hline Variable & $\boldsymbol{P}$ & $\boldsymbol{T}$ & $\boldsymbol{C}$ & Efficiency \\
\hline Reference system (Software) & 2297.26 & 300.00 & 127.00 & $100.00 \%$ \\
\hline Examined system (Human) & 420.00 & 300.00 & 12.90 & $55.60 \%$ \\
\hline Examined system (Human, more time) & 2297.26 & 300.00 & 18.95 & $14.92 \%$ \\
\hline
\end{tabular}

As it can be clearly seen in the results, software clash detection is more efficient, as was expected. Also there is clear decline in the efficiency of the human factor, correlating with design complexity, as it can be seen, when Project $X$ and Project $Y$ efficiency is compared. In the presented projects, it is also safe to say that more time for the examination by experts does not result in better efficiency, although more total clashes were found. There is probably a limit to how many clashes are even identifiable by human experts.

\section{Discussion}

It was shown that benefits of using BIM for clash detection can be used to enhance the efficiency of construction management and to generate huge savings by early collision prevention. This is true especially in construction of industrial complexes, which are often built in rural environment. However, it is necessary to ensure an appropriate coordination among stakeholders. It is important to maintain BIM model including unifying individual model libraries and definitions. When properly used, the clash detection system should provide significant savings. Managers should therefore ensure good workflow, delegate capable workers to process models and establish a system of retrospective changing the design and general responses based on the results of clash detection. The problem might be relatively higher starting investment to BIM tools and staff training. Cost saving should be far higher than the mentioned investments at the end. However, the question is how large the project must be to generate such savings. This exceeds the scope of this paper and therefore it is an opportunity for further research.

As disputes [11] and coordination problems [12] are often considered as one of the most critical cause of low performance in construction projects, clash detection is extremely important to avoid such cases. This is most important in the beginning phases of construction projects, even during architectural studies [13]. The future question is how various software tools will be able to react on increasing the demand for such functionality [14]. It is also important to define process standards for proper clash detection implementation [15], as if this is done in later phases, it may result in failure [16] and possible issues in the operational phase.

The clash detection process is important for most types of the projects. Even though it might seem that this tool is better suited for building structures, transport infrastructure projects often have highdensity network of lines and costs related to changes, when clashes are not avoided, are very high, too [17]. Although the supply system might play a role in evaluating the efficiency of clash-detection tools, it is not that relevant whether the clashes are detected in between the project partners or if the process of clash detection is integrated into the agenda of one of the project participants. 
The proposed P-T-C analysis is applicable, as it was shown on a practical example. Although the presented examples are not statistically relevant (more tests should be conducted with different variables and project types), they clearly demonstrate the P-T-C analysis simplicity and possible use.

\section{Conclusions}

This paper described a definition of a clash along with its origin in computer games and object, volume modeling. Built environment has implemented this method in the control system through the functions of BIM software. It was emphasized that despite of indisputable potential benefits of using BIM, it is still necessary to ensure convenient supervision. Simple clashes taxonomy was proposed. Clashes have been sorted in four categories: Hard clashes, Soft clashes, $4 D$ clashes and Animated clashes. Every category has been explained. The importance of clashes was described in the third part of this paper. According to cited sources, the clashes represent potential threats. They may cause high expenditures, significant delays, injuries and even deaths. Processes of clash resolving were described in the next part concerning different methods and approaches. In the last part of the paper, a very simple methodology (P-T-C analysis) for quantifying the efficiency of computable clash detection was introduced and explained on practical examples with conclusions. These case studies are not statistically relevant and were merely conducted as a way to introduce the proposed formulas.

\section{Acknowledgements}

This work was supported by the Grant Agency of the Czech Technical University in Prague, grant No. SGS17/121/OHK1/2T/11.

\section{References}

[1] Obi I. What is clash detection? How does BIM help? 2016 [online] [2016-08-20]. Available at: http://www.thebimcenter.com/2016/03/what-is-clash-detection-how-does-bim-help.html

[2] Hi-Tech CADD Services. 3 Types Of 3D BIM Clash Detection Have Their Own Importance. 2014 [online] [2016-11-02]. Available at: http://www.hitechcaddservices.com/news/3-types-of3d-clash-detection-have-their-own-importance/

[3] Reddy P. BIM for building owners and developers: Making a business case for using BIM on projects. Hoboken, New Jersey: John Wiley \& Sons, Inc., 2012. ISBN 978-0-470-90598-2.

[4] Kymmel W. Building Information Modelling: Planning and Managing Construction Projects with 4D CAD. New York: The McGraw-Hill Companies, Inc., 2008. ISBN 0-07-149453-7.

[5] Hi-Tech CADD Services. BIM for Clash Detection and Risk Analysis Streamlines the Construction Process. 2014 [online] [2016-11-18]Available at: http://www.hitechcaddservices.com/news/bim-for-clash-detection-and-risk-analysis-streamlinesthe-construction-process/

[6] The Waste and Resources Action Programme. Case study: Achieving resource efficiency though BIM. In: The Waste and Resources Action Programme. 2013 [online] [2016-12-01]. Available at: http://www.wrap.org.uk/sites/files/wrap/Leeds_Arena_FINAL.pdf

[7] Lefevre M. Build Smarter, Faster, and Cheaper with BIM. 2008 [online] [2016-12-05]. Available at: http://www.buildings.com/article-details/articleid/6149/title/build-smarter-faster-and-cheaperwith-bim

[8] Tesseract-Design. Web Tesseract Design. BIM Coordination During Design: Do It Right the First Time - Using BIM to Coordinate Systems. 2014 [online] [2016-12-06]. Available at: http://www.tesseract-design.com/bim-coordination-during-design.html

[9] Redpoint Positioning Corporation. Manage Construction Operations in Real Time. Redpoint Positioning. 2015 [online][2016-11-14]. Available at: https://www.redpointpositioning.com/rtlsfor-vertical-industries/rtls-for-construction/

[10]Eastman C., Teicholz P., Sacks R. a Liston K. BIM Handbook: A Guide to Building Information Modeling for Owners, Managers, Designers, Engineers and Contractors. $2^{\text {nd }}$ Edition. John Wiley \& Sons, Inc., 2011. ISBN 978-04-7054-137-1.

[11] Charehzehi A., Chai C., MdYusof A., Chong H.-Y., Loo S.C. Building information modeling in construction conflict management. International Journal of Engineering Business Management, vol. 9, 2017. 
[12] Akponeware, A.O., Adamu, Z.A. Clash detection or clash avoidance? An investigation into coordination problems in 3D BIM. Buildings, vol. 7(3), 2017, art.no. 75.

[13] Bouška, R., Heralová, R.S. Utilization Of BIM during architectural study. CESB 2016 - Central Europe Towards Sustainable Building 2016: Innovations for Sustainable Future, vol. 2, 2016, pp. 677-684.

[14]Bouška R. Evaluation of Maturity of BIM Tools across Different Software Platforms. Procedia Engineering, vol. 164, 2016, pp. 481-486.

[15] Kvirenc P., Bouska R. Case study: BIM protocol for Skanska (Czech Republic). Business \& IT, vol. VII(1), 2017, pp. 8-13

[16] Matějka P., Kosina V., Tomek A., Tomek R., Berka V., Šulc D. The Integration of BIM in Later Project Life Cycle Phases in Unprepared Environment from FM Perspective. Procedia Engineering, 164, 2016, pp. 550-557.

[17] Tomek R., Vitásek S. Improvement of economic effectiveness of road highway projects, Procedia Engineering, 164, 2016, pp. 395-401. 